\title{
IOT based Automation for Smart Sustainable Homes
}

\author{
Atif Abdus Samad ${ }^{1}$, Farheen Siddiqui ${ }^{2}$ \\ \{atifsamad30@gmail.com, fsiddiqui@jamiahamdard.ac.in\} \\ ${ }^{1,2}$ Department of CSE, SEST, Jamia Hamdard, India
}

\begin{abstract}
This paper proposes the design of Inter of Things (IoT) based home automation system using Raspberry pi. Unlike the conventional fixed switches for switching on and off- fans, bulbs and other electrical devices, it would provide users portable control and management all the devices connected together over wireless local area network. Moreover this paper lays emphasis on the security of the user's home by providing biometric door lock/unlock feature through fingerprint sensor of a smart phone. This paper also include a water tank management system with objective to send notifications to the user about water level with option of automatically cutting off the power supply at full tank level thereby preventing any water wastage. The main objective is to provide the users with a simple graphical user interface in form of a mobile application that will allow them to control and automate their household devices, provide them with security features and prevent wastage of natural resources like water and electricity with just a simple touch on their smart phone which eventually make smart homes sustainable too.
\end{abstract}

Keywords: IOT, smart home, raspberry pi,Android

\section{Introduction}

\begin{abstract}
Almost everyone today owns a smart phone; it has become a part of our day to day life. We can make use of our very own smart phone for operating and controlling household devices and appliances with all advanced features such as a powerful processor and sensors like fingerprint sensor etc. instead of using the conventional on/off wall mount switches, at our own comfort within a wide range area covered by the Wi-Fi network which can be further expanded by using repeaters if required, thereby increasing the accessibility in addition to the ease of use.
\end{abstract}

Using the android application a user can set an on-off timer for a device of his choice, for example if the user want that a particular bulb should light-up at $7 \mathrm{pm}$ without his direct intervention, he can set a timer in the app that would send the signal at the directed time to the pi module to switch on the bulb. 
This paper not only provides a smart and easy way to control devices it also adds on to the security features of the house, by replacing the conventional door lock with a solenoid door lock which can read input signals from the pi module, the user would be able to unlock the door by placing his finger on the fingerprint scanner of his mobile phone, which verifies the data with the pre- scanned and stored fingerprint of the owner and unlocks the door if a match is found.

\section{Related Work}

IOT is the buzzword that has promised to deliver its immense benefits for societal applications [3]. IoT has been centre of focus especially for scientists, industry and government worldwide due to its promises towards revolutionary changes in human life. IoT is a technology that is deep rooted as billions of sensors that are connected to the World Wide Web via wireless and other communication technologies. The sensors are capable of generating huge volumes of data. This generated data can be analysed, interpreted through different approaches and then utilized for various applications [2]. The concept of automating homes is based on underlying synergy of of interconnected home appliances through internet (IOT) and Raspberry pi $[5,6]$. This is for the purpose of remote controlling, operation and monitoring of the almost all devices in a home that are on network (electricity operated or electronically functional) from any general location. Remote control signal can be send from any device at remote location such as a Smartphone. There are available implementations of a home automation system that is flexible as per user requirement and low in cost too. These system increases the usage of wireless mode of communication providing the user with remote control of various home appliances operated through electricity [4]. Shaiju et al's work is based on the same concept that is presented in this paper. In their work a HAS (Home Automation System) is proposed. The proposed work uses the concept of connected home appliances through Raspberry Pi . This connection is done using relay circuit. User interface for controlling devices is implemented through an android application.[6, 7]. Home automation using Raspberry Pi [8] has used a Raspberry pi model B as a processing unit. For internet connectivity a Wi-Fi dongle is employed and connection of home appliances is established through a relay circuit. A WebIOPi framework is referred for IOT framework..[9]

\section{Methodology}

From the End user's perspective, the system should is easy to understand and access without much technical knowledge so that any person can use it easily and establish a remote connection with the raspberry pi over a Wi-Fi network 
using socket programming, thus the mobile application provides a material designed simple and easy to use graphical user interface with different activities to control and operate different devices attached to the pi module.

\section{a. User Classes and Characteristics}

The user can be divided into two main classes :

i. The Admin: They manage the entire system. They act as an intermediate between mobile application and the end devices such as bulb, fan, door lock etc. The admin is responsible for turning off/on the end devices by sending the control signals from the android application to server and finally to the end devices.

ii. The End user: The one who benefits from the automation of the end devices by this system, the end user includes the admin himself/herself.

\section{b. Assumptions and Dependencies}

- The user should have knowledge of Wi-Fi network connection and Android Device.

- The system depends on a Wi-Fi connection, both the android device as well as the pi module must be connected to the same Wi-Fi network.

- The range of the system depends upon the range of the Wi-Fi network and can be increased by using repeater if required.

- Roles and tasks are predefined.

\section{c. System Design}

The design of IOT in Home Automation broadly has two parts (Fig 1),

i. Android application and

ii. Raspberry pi module

The android application is developed for this paper is based on stack- Java for the programming part, Xml for Layout Design and Android Studio and Android Developer Tools as the toolset and Integrated Development Environment (Fig 1). 
All the devices to be controlled and automated by the user using the android application are connected to the general purpose input/output pins of the raspberry (Fig 2) and are provided with a power source either from the pi module which can provide a power supply of up to 5 volts or they can be connected to an external power source. There are a total of forty pins on the module of which twenty eight are GPIO and rest are reserved for advance use. The GPIO (general purpose input/output) pins are a powerful feature of pi module as any of them can be assigned in software as input or output pin and used for wide range of applications. These GPIO pins can be set either to high (3V3) or low (0V), number of programming languages like Scratch 1.4, Scratch 2, Python etc. could be used these GPIO pins. In this paper we have used Python as the programming language to control these pins and the functioning of the devices connected to these pins.

A socket is a way that provides communication medium between two ends using TCP (Transmission Control Protocol), these two ends are server and the client end. In this paper raspberry pi and the android application are the two ends or terminals that communicate to each other over a Wi-Fi network using TCP (Transmission Control Protocol). The android application serves as the client side where the user has a material designed and easy to use interface with options of selecting the device to control and regulate with a touch on his device, this touch on the mobile device is captured and a signal relating to the selected device is send to raspberry pi which acts as the server, reads the byte stream encoded in UTF-8 format sent from the android application decodes it and generates an output signal for the particular device connected to raspberry pi through GPIO, this output signal is either high or low depending upon the data received from the android application. In this way the two terminals communicate to each other. The range of communication between the two ends that is the android application and the pi module depends on the range of the Wi-Fi network and can be increased by using repeaters if required. The two ends will continue to communicate smoothly till they are connected to the same Wi-Fi network. The android application also requires the user to mention the ip address and the port number of the raspberry pi module in order to establish the connection between them over the Wi-Fi network using TCP.

The android application uses the internet permission and the fingerprint senor permission in order to connect to a wifi network and to read and verify user's fingerprint when sending signal for biometric door unlock system(Fig 3)). The 
android application has multiple activities including the splash screen, home page and various individual activities for different devices connected to the pi module. The splash screen is just a display of this papers title and a small animation of what the user is about to do with this android app. The home page has the title bas with the app name, an expandable image view, an input text field which takes the IP address and the port number of the raspberry pi as input from the user on which to establish the connection. Then the screen shows icon and labels of the devices and systems that could be controlled using this android application. As soon as the user clicks on the labelled icon of the device to control its working the android application takes the user to a new activity relating to the operations of that particular device and send a signal to raspberry pi indicating that a particular device has been selected by the user to perform operation upon. This screen has an image view and buttons for basic operations of the selected device, as soon as a button is clicked a signal is triggered and send to pi module to perform the selected operation on the device and the image view also changes, representing the current state of that device. 


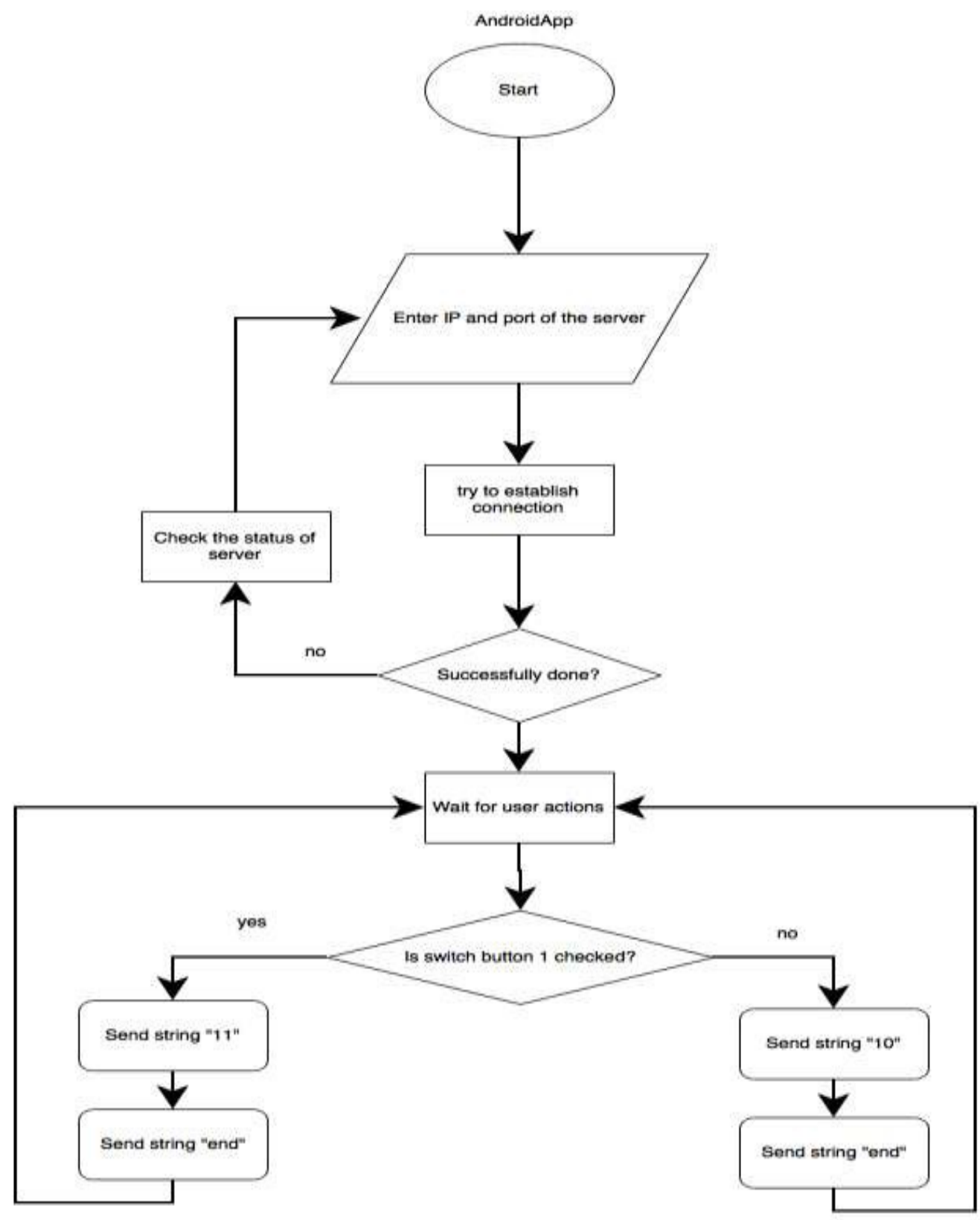

Fig 1: Flowchart for Android Application 


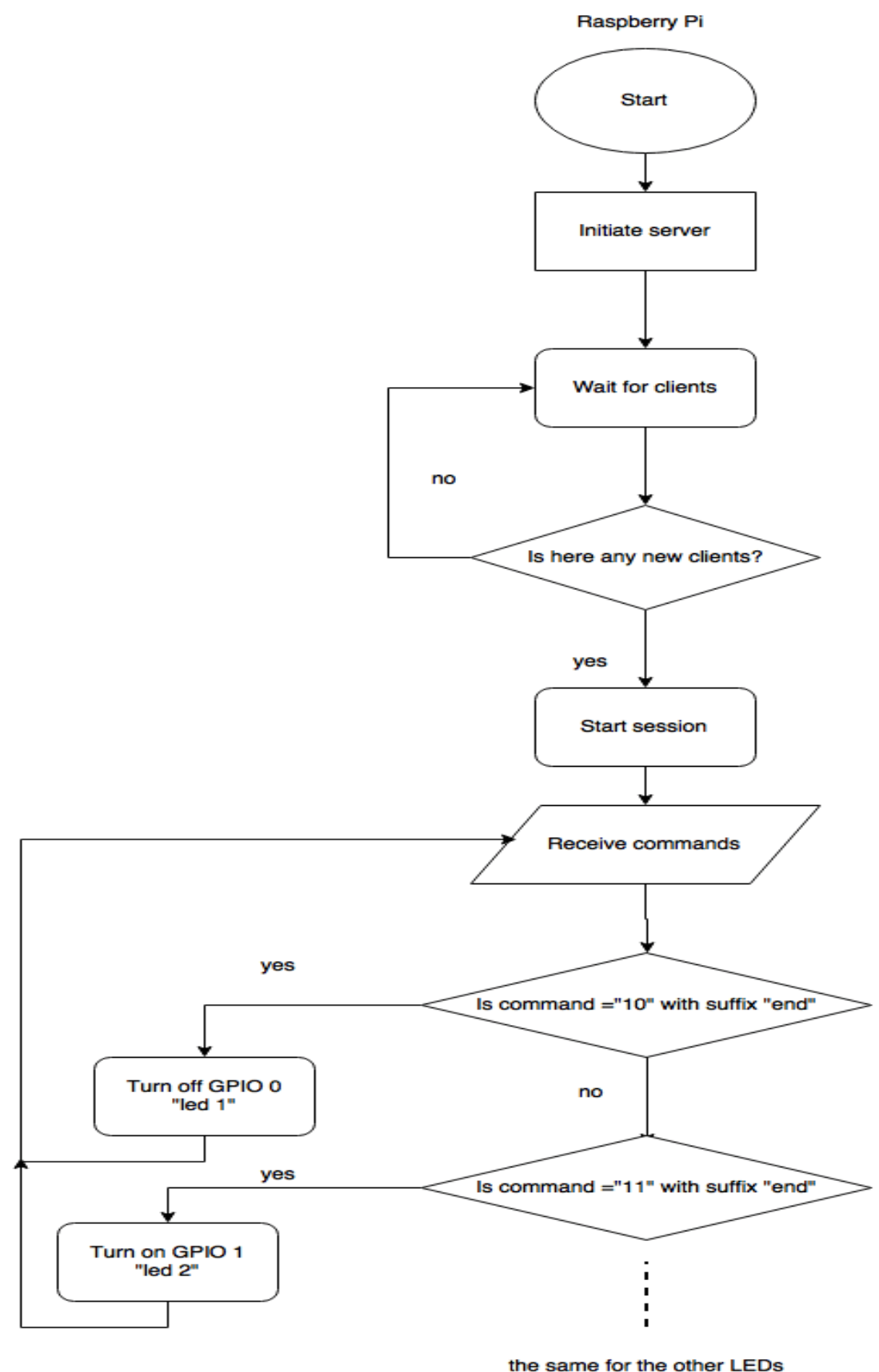

Fig 2: Flowchart for raspberry pi module 
On the Server side, the system is used to send signals from the raspberry pi to all the devices attached to the pi. The server side listen for an active connection on a particular port number of the pi, as soon as a request is send from the android device a connection is established and the server that is the pi module wait for control signals from the android application which are received over TCP and further transmitted to the end devices.

From the End user's perspective, the system should is easy to understand and access without much technical knowledge so that any person can use it easily and establish a remote connection with the raspberry pi over a Wi-Fi network using socket programming, thus the mobile application provides a material designed simple and easy to use graphical user interface with different activities to control and operate different devices attached to the pi module.(Fig 4). This design has following limitations

- The range of the system is limited to the range of the Wi-Fi network.

- Both the terminals that is the server and the client needs to be connected to the same Wi-Fi network.

- The smartphone needs to have a fingerprint scanner.

- The user should have basic knowledge of Wi-Fi connectivity such as knowing the IP address of the server and the android applications.

- Internet connectivity is required.

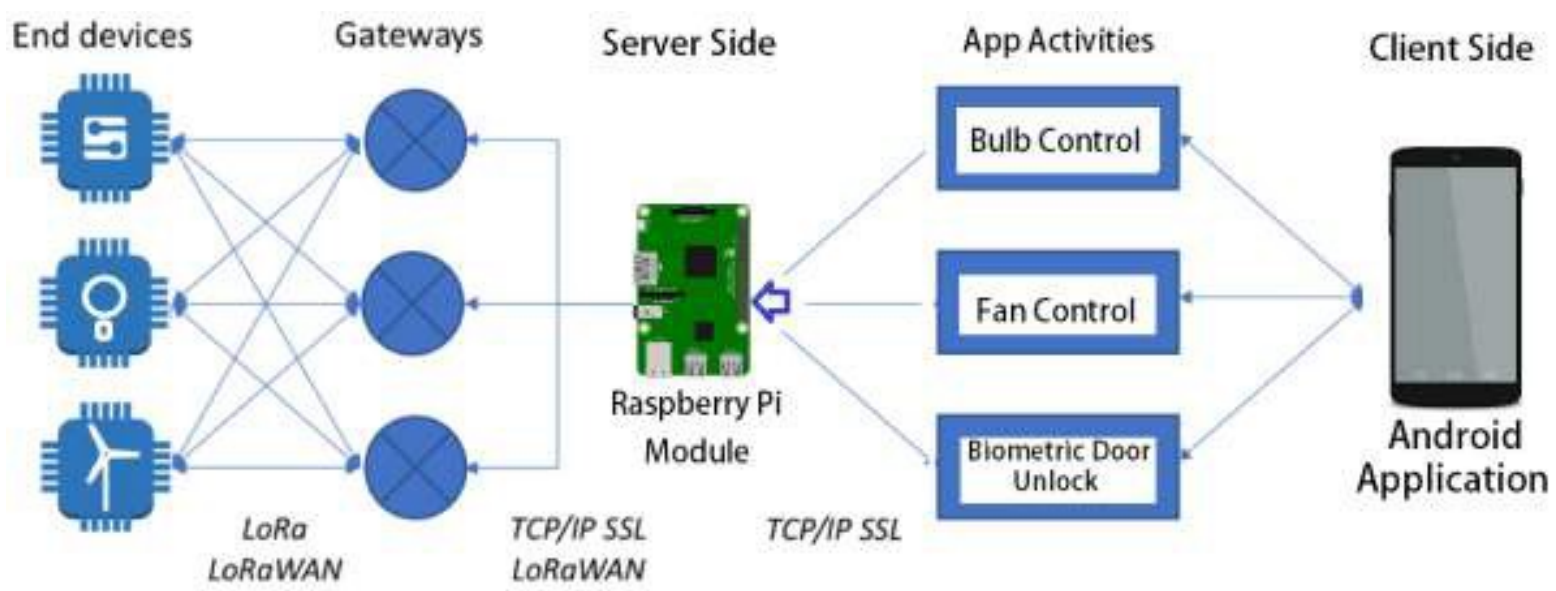

Fig 3: Home automation Architecture 


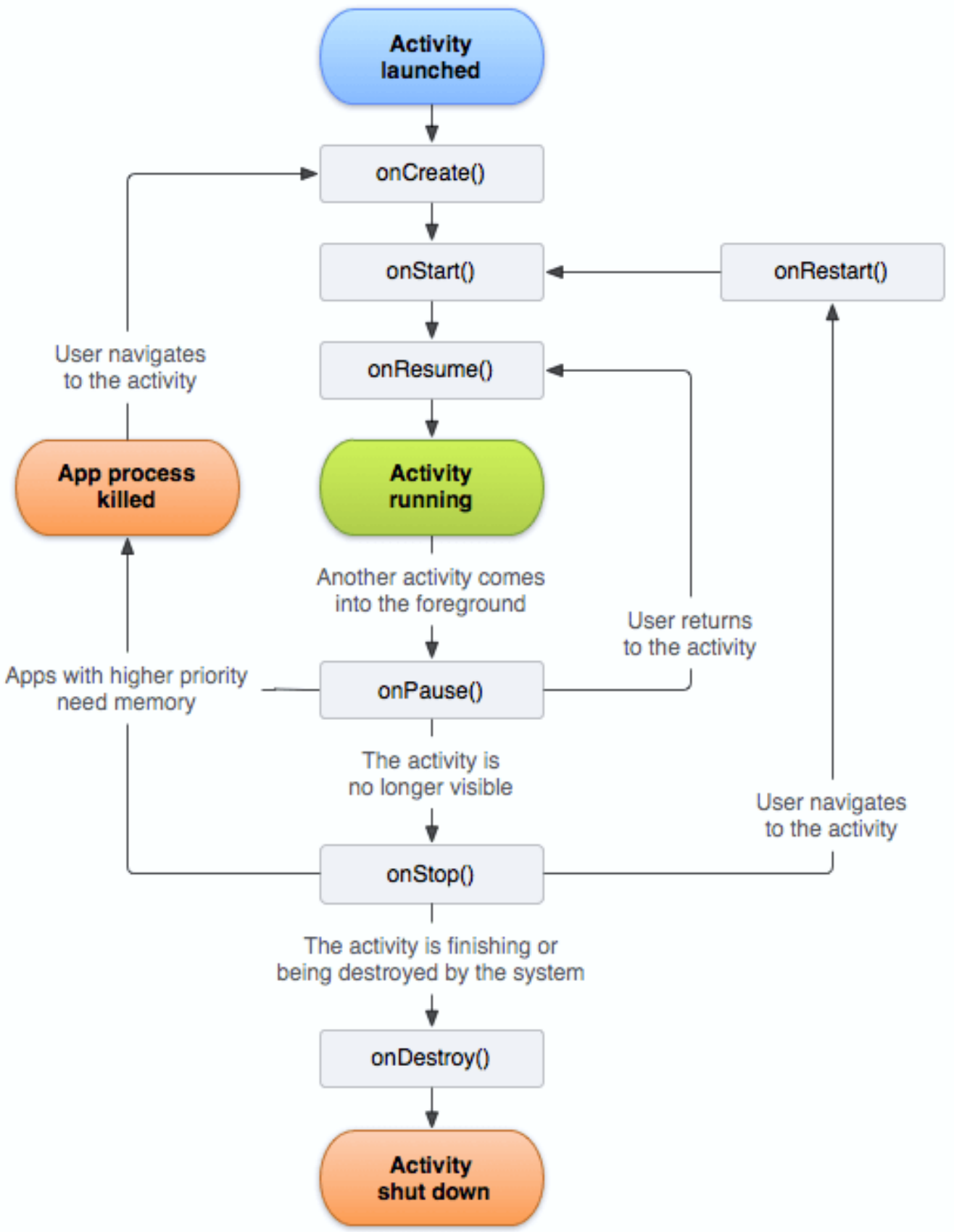

Fig 4: Android application activity diagram 


\section{Experiments}

The proposed work is implemented and all the remote controlling is experimented using android frontend. Fig 4 shows the screen presenting the options of device control to the user. Fig 5 shows option for controlling a home bulb from a remote location and similarly Fig 6 and Fig 7 shows android application screenshots for Fan remote control and security using biometric lock.

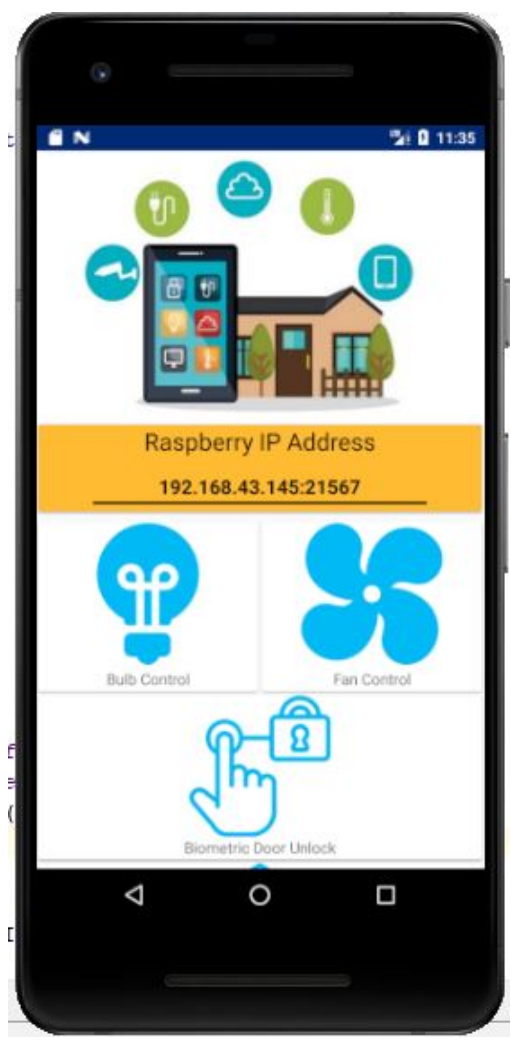

Fig 5: Main screen

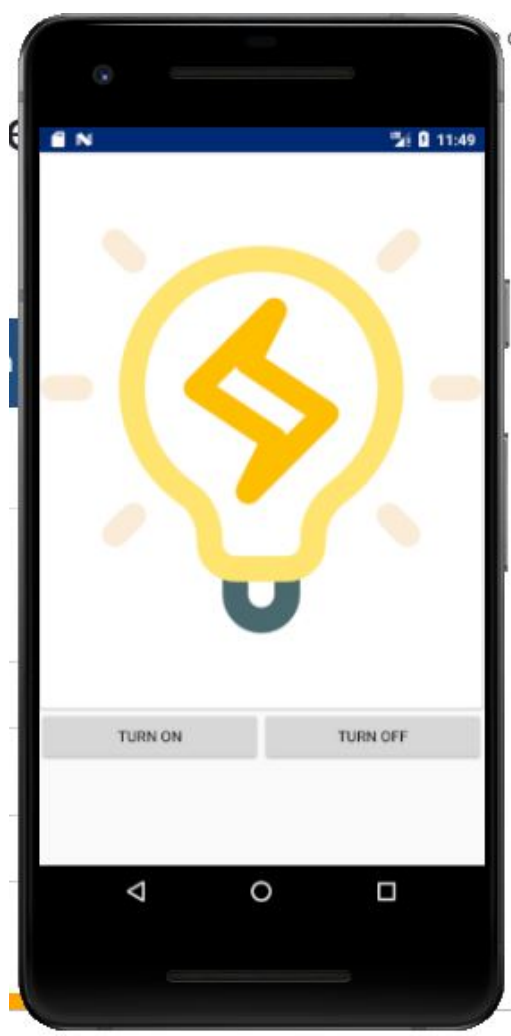

Fig 6: Bulb control 


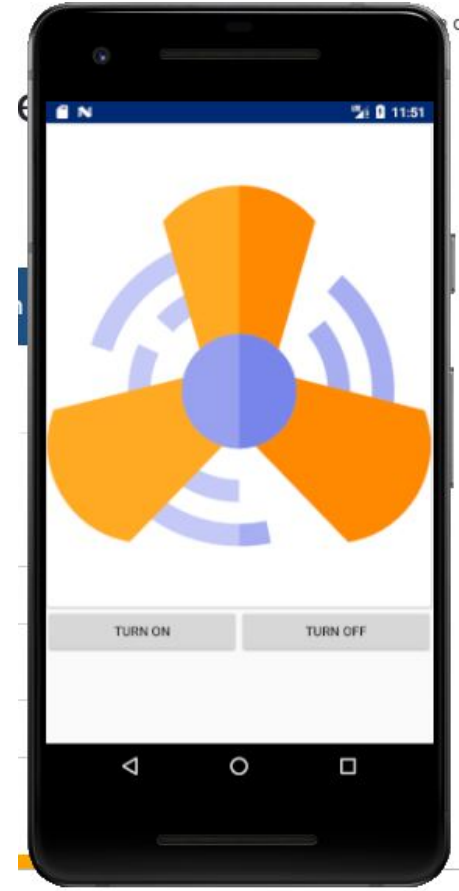

Fig 7: Fan control option

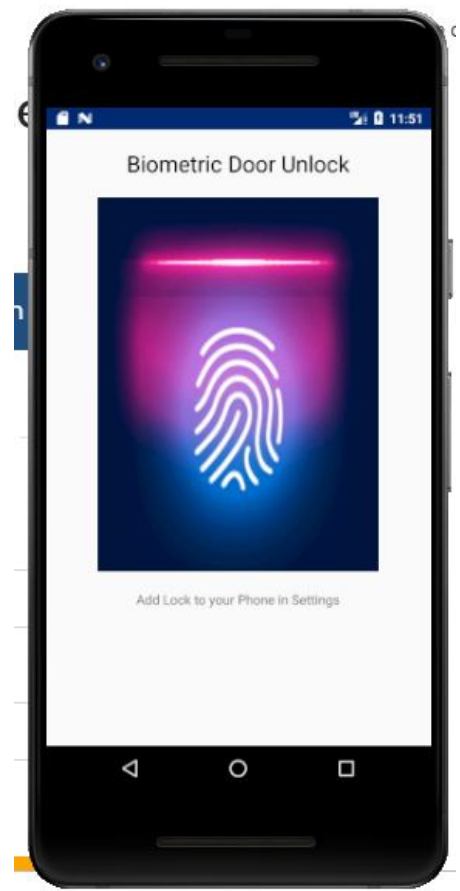

Fig 8: Lock option

\section{Conclusion}

The paper "Application of IOT in Home Automation" has been developed to implement IOT in our household and make it easier and smarter for the users to manage and control devices such as bulbs, fans etc. from any location within the Wi-Fi network range at the click of a button on his smartphone. The paper also intends to add on security features to one's home by providing features like fingerprint scan for unlocking the door, thereby providing security in addition to ease of use. It also lays emphasis on saving water by providing an auto cut mechanism. All the requirement specifications were followed as far as possible and few additional features were added that can make it more user- friendly.Together these features save energy and water that converts simple home into sustainable home. Large percentages of population worldwide do not have access to safe and managed connection of water. Consequently they are also deprived of proper sanitation facilities. Scarcity of water at one place and flooding at another is due to improper water management. This is a major challenge in social and economic development. As per goal 6 for sustainable development, bringing up the scale of water efficiency and getting better water management are key to balancing the challenging and emergent water supply 
difficulty from different sectors and users.

\section{References}

[1] D. Norris, The Internet of Things: Do-It-Yourself at Home Projects for Arduino, Raspberry Pi and BeagleBone Black. Tab Electronics, 2015.

[2] D. Giusto, A. Iera, G. Morabito, and L. Atzori, The Internet of Things. New York, NY: Springer New York, 2010.

[3] Raspberry pi as a sensor Web node for home automation Vladimir Vujovic, Mirjana Maksimovic http://dx.doi.org/10.1016/j.compeleceng.2015.01 .019

[4] A. Z. Alkar and U. Buhur, "An internet based wireless home automation system for multifunctional devices,” IEEE Trans. Consum. Electron., vol. 51, no. 4, pp. 1169-1174, Nov. 2005.

[5] Jump up Bush, Steve (25 May 2011). "Dongle computer lets kids discover programming on a TV". Electronics Weekly. Retrieved 11 July, 2011

[6] Shaijupaul, Ashlin Anthony, Aswathy B, "Android based Home Automation Using Raspberry $\mathrm{Pi}$, IJCAT - International Journal of Computing and Technology, Volume 1,Issue 1, February 2014, pp 143

$-147$

[7] Pawan singh,Krupachotalia,SanketPingle, " Review paper on SmartGSM based Home Automation System".International Research Journal of Engineering and Technology (IRJET), Volume: 3, Issue :04 April - 2016, pp 1838 - 1843.

[8] Monika M Patel, Mehul A Jajal, Dixita B vataliya, "Home automation using Raspberry Pi, "International Journal of Innovative and Emerging Research in Engineering Volume 2, Issue 3, 2015

[9] Monika M Patel, Mehul A Jajal, Dixita B vataliya, "Home automation using Raspberry Pi, "International Journal of Innovative and Emerging Research in Engineering Volume 2, Issue 3, 2015. 